\title{
Exact solutions for differential-algebraic equations
}

\author{
Hector Vázquez-Leal
}




\title{
EXACT SOLUTIONS FOR DIFFERENTIAL-ALGEBRAIC EQUATIONS
}

\author{
HECTOR VAZQUEZ-LEAL
}

Received 22 October, 2013

\begin{abstract}
This work presents the application of series method to find solutions of differentialalgebraic equations systems (DAEs). We present two case studies to show that series method generates approximate solutions for DAEs. The type of tested equations are a linear and a nonlinear DAEs of index-3. What is more, we present the post-processing of the series solutions with Laplace-Padé (LP) resummation method as a useful strategy to find exact solutions or to extend the domain of convergence of the power series solutions.
\end{abstract}

2010 Mathematics Subject Classification: 34L30

Keywords: series method, resummation methods, differential-algebraic equations

\section{INTRODUCTION}

Solving differential equations is an important issue in sciences because many physical phenomena are modelled using such equations. Modern methods like homotopy perturbation method (HPM) [7, 8, 14, 16, 22, 23, 35, 41-43], homotopy analysis method (HAM ) [13, 15,32], variational iteration method (VIM) [1,5,20], among others, are powerful tools to approximate nonlinear dynamic problems. Nevertheless, series method $[9,10,17]$ is a well known classic procedure from literature that can be applied successfully to solve differential equations. This method establishes that the solution of a differential equation can be expressed as a power series of the independent variable. Therefore, in this work, we apply series method to solve two differential-algebraic equations. Additionally, we present the use of Laplace-Padé (LP) resummation method as an useful strategy to obtain exact solutions or approximations possessing a large domain of convergence.

This paper is organized as follows. In Section 2, we introduce the basic concept of the series method. The concept about Laplace-Padé resummation method is explained in Section 3. The solution of two differential algebraic equations is presented

I gratefully acknowledge the financial support of the National Council for Science and Technology of Mexico (CONACyT) through grant CB-2010-01 \#157024. 
in Section 4. In Section 5, numerical simulations and a discussion about the results are provided. Finally, a brief conclusion is given in Section 6.

\section{BASIC CONCEPT OF SERIES METHOD}

It can be considered that a nonlinear differential equation can be expressed as

$$
A(u)-f(t)=0, \quad t \in \Omega,
$$

having as boundary condition

$$
B\left(u, \frac{\partial u}{\partial \eta}\right)=0, \quad t \in \Gamma,
$$

where $A$ is a general operator, $f(t)$ is a known analytic function, $B$ is a boundary operator, and $\Gamma$ is the boundary of domain $\Omega$.

The series method establishes that the solution of a differential equation can be written as

$$
u=\sum_{i=0}^{\infty} u_{i} t^{i},
$$

where $u_{0}, u_{1}, \ldots$ are unknowns to be determined by series method.

The basic process of series method can be described as:

(1) Equation (2.3) is substituted into (2.1), then we regroup equation in terms of $t$-powers.

(2) We equate each coefficient of the resulting polynomial to zero.

(3) The boundary conditions of (2.1) are substituted into (2.3) to generate an equation for each boundary condition.

(4) Aforementioned steps generate a nonlinear algebraic equation system (NAEs) in terms of the unknowns of (2.3).

(5) Finally, we solve the NAEs to obtain $u_{0}, u_{1}, \ldots$, coefficients.

\section{LAPLACE-PADÉ RESUMMATION METHOD}

Several approximate methods provide power series solutions (polynomial). Nevertheless, sometimes, this type of solutions lacks of large domains of convergence. Therefore, Laplace-Padé [3, 6, 11, 18, 21, 26-28, 31, 33] resummation method is used in literature to enlarge the domain of convergence of solutions or inclusive to find exact solutions.

The Laplace-Padé method can be explained as follows:

(1) First, Laplace transformation is applied to power series (2.3).

(2) Next, $s$ is substituted by $1 / t$ in the resulting equation.

(3) After that, we convert the transformed series into a meromorphic function by forming its Padé approximant of order $[N / M]$. $N$ and $M$ are arbitrarily chosen, but they should be of smaller value than the order of the power series. 
In this step, the Padé approximant extends the domain of the truncated series solution to obtain better accuracy and convergence.

(4) Then $t$ is substituted by $1 / s$.

(5) Finally, by using the inverse Laplace $s$ transformation, we obtain the exact or approximate solution.

This process is known as Laplace-Padé series method (LPSM).

\section{Case Studies}

In this section we will solve two DAE problems in order to depict the LPSM method.

4.1. Hessenberg index-3 DAE

Consider the following DAE [34]

$$
\begin{aligned}
x_{1}^{\prime}+x_{1}-t x_{3}+x_{4} & =0, \\
x_{2}^{\prime}-x_{1}+x_{2}-t^{2} x_{3}+t x_{4} & =0, \\
x_{3}^{\prime}-t^{3} x_{1}+t^{2} x_{2}-x_{3} & =0, \\
t x_{1}-x_{2}+t x_{3}-x_{4} & =0, \\
x_{1}(0)=x_{3}(0) & =1, \\
x_{2}(0)=x_{4}(0) & =0,
\end{aligned}
$$

where prime denotes derivative with respect to $t$.

We suppose that solution for (4.1) has the following fourth order expression

$$
\begin{array}{ll}
x_{1}(t)=\sum_{i=0}^{4} x_{1 i} t^{i}, & x_{2}(t)=\sum_{i=0}^{4} x_{2 i} t^{i}, \\
x_{3}(t)=\sum_{i=0}^{4} x_{3 i} t^{i}, & x_{4}(t)=\sum_{i=0}^{4} x_{4 i} t^{i},
\end{array}
$$

Substituting (4.2) into (4.1), rearranging and equating terms having the same $t$-powers, we obtain

$$
\begin{array}{r}
x_{11}+x_{40}+x_{10}+\left(x_{41}+x_{11}+2 x_{12}-x_{30}\right) t+\cdots=0 . \\
x_{21}+x_{20}-x_{10}+\left(x_{40}+2 x_{22}+x_{21}-x_{11}\right) t+\cdots=0 . \\
x_{31}-x_{30}+\left(-x_{31}+2 x_{32}\right) t+\cdots=0 .
\end{array}
$$




$$
-x_{20}-x_{40}+\left(x_{10}-x_{41}-x_{21}+x_{30}\right) t+\cdots=0 .
$$

Next, equating the coefficients of (4.3) to zero, we obtain the following system of algebraic equations

$$
\begin{aligned}
& t^{0}: x_{11}+x_{40}+x_{10}=0, \\
& t^{1}: x_{41}+x_{11}+2 x_{12}-x_{30}=0, \\
& \quad \vdots \\
& t^{3}: \cdots \\
& t^{0}: x_{21}+x_{20}-x_{10}=0, \\
& t^{1}: x_{40}+2 x_{22}+x_{21}-x_{11}=0, \\
& \quad \vdots \\
& t^{3}: \cdots \\
& t^{0}: x_{31}-x_{30}=0, \\
& t^{1}:-x_{31}+2 x_{32}=0, \\
& \quad \vdots \\
& t^{3}: \cdots \\
& t^{0}:-x_{20}-x_{40}=0, \\
& t^{1}: x_{10}-x_{41}-x_{21}+x_{30}=0, \\
& \quad \vdots \\
& t^{4}:-x_{24}+x_{33}+x_{13}-x_{44}=0
\end{aligned}
$$

Now, in order to consider the initial condition of (4.1), we substitute them into (4.2) to obtain

$$
\begin{array}{ll}
x_{10}=1, & x_{20}=0, \\
x_{30}=1, & x_{40}=0,
\end{array}
$$

It is important to notice that, from (4.4)-(4.6), we use only the powers $t^{i}(i=0,1,2,3)$, because the rest of the information needed is taken from (4.8). From (4.1), we can observe that there is not an explicit equation for variable $x_{4}$. Therefore, from (4.7), 
we use coefficients of powers $t^{i}(i=1,2,3,4)$ to collect enough information to compensate the presence of $x_{4}$ in those equations. Furthermore, order zero term of (4.7) possesses redundant information that can be ignored. Hence, for (4.7), we can use powers $t^{i}(i=1,2,3,4)$. Then, solving the NAEs composed by (4.4), (4.5), (4.6), (4.7), and (4.8) results the following approximate solution

$$
\begin{aligned}
& x_{1}(t)=1-t+\frac{1}{2} t^{2}-\frac{1}{6} t^{3}+\frac{1}{24} t^{4}, \\
& x_{2}(t)=t-t^{2}+\frac{1}{2} t^{3}-\frac{1}{6} t^{4}, \\
& x_{3}(t)=1+t+\frac{1}{2} t^{2}+\frac{1}{6} t^{3}+\frac{1}{24} t^{4}, \\
& x_{4}(t)=t+t^{2}+\frac{1}{2} t^{3}+\frac{1}{6} t^{4} .
\end{aligned}
$$

Then, Laplace transformation is applied to (4.9) and then $1 / t$ is written in place of $s$. Afterwards, Padé approximant of order [2/2] is applied and $1 / s$ is written in place of $t$ for each variable. Finally, by using the inverse Laplace $s$ transformation, we obtain the exact solution for (4.1)

$$
\begin{aligned}
& x_{1}(t)=\exp (-t), \\
& x_{2}(t)=t \exp (-t), \\
& x_{3}(t)=\exp (t), \\
& x_{4}(t)=t \exp (t) .
\end{aligned}
$$

\subsection{Index-three nonlinear differential-algebraic equation system}

Consider the following nonlinear DAE [2]

$$
\begin{aligned}
y_{1}^{\prime} & =2 y_{1} y_{2} z_{1} z_{2}, \\
y_{2}^{\prime} & =-y_{1} y_{2} z_{2}^{2}, \\
z_{1}^{\prime} & =\left(y_{1} y_{2}+z_{1} z_{2}\right) u, \\
z_{2}^{\prime} & =-y_{1} y_{2}^{2} z_{2}^{2} u, \\
y_{1} y_{2}^{2} & =1, \\
y_{1}(0) & =y_{2}(0)=1, \\
z_{1}(0) & =z_{2}(0)=1, \\
u(0) & =1,
\end{aligned}
$$

where prime denotes derivative with respect to $t$. 
We suppose that solution for (4.11) has the following fourth order expression

$$
\begin{array}{rlrl}
y_{1}(t) & =\sum_{i=0}^{4} y_{1 i} t^{i}, & y_{2}(t) & =\sum_{i=0}^{4} y_{2 i} t^{i}, \\
z_{1}(t) & =\sum_{i=0}^{4} z_{1 i} t^{i}, & z_{2}(t) & =\sum_{i=0}^{4} z_{2 i} t^{i}, \\
u(t) & =\sum_{i=0}^{4} u_{i} t^{i} . &
\end{array}
$$

Substituting (4.12) into (4.11), rearranging and equating terms having the same $t$ powers, we obtain

$$
\begin{aligned}
& y_{11}-2 y_{10} y_{20} z_{10} z_{20}+\left(-2 y_{10} y_{20} z_{11} z_{20}+2 y_{12}-2 y_{10} y_{21} z_{10} z_{20}\right. \\
& \left.-2 y_{11} y_{20} z_{10} z_{20}-2 y_{10} y_{20} z_{10} z_{21}\right) t+\cdots=0 . \\
& y_{21}+y_{10} y_{20} z_{20}^{2}+\left(y_{10} y_{21} z_{20}^{2}+2 y_{2} 2+2 y_{10} y_{20} z_{20} z_{21}+y_{11} y_{20} z_{20}^{2}\right) t+\cdots=0 . \\
& z_{11}-u_{0} y_{10} y_{20}-u_{0} z_{10} z_{20}+\left(-u_{0} y_{10} y_{21}-u_{1} z_{10} z_{20}\right. \\
& \left.-u_{0} y_{11} y_{20}-u_{0} z_{10} z_{21}-u_{0} z_{11} z_{20}-u_{1} y_{10} y_{20}+2 z_{12}\right) t+\cdots=0 . \\
& y_{10} y_{20}^{2} z_{20}^{2} u_{0}+z_{21}+\left(2 z_{22}+2 y_{10} y_{20}^{2} z_{20} z_{21} u_{0}\right. \\
& \left.+y_{11} y_{20}^{2} z_{20}^{2} u_{0}+y_{10} y_{20}^{2} z_{20}^{2} u_{1}+2 y_{10} y_{20} y_{21} z_{20}^{2} u_{0}\right) t+\cdots=0 . \\
& -1+y_{10} y_{20}^{2}+\left(y_{11} y_{20}^{2}+2 y_{10} y_{20} y_{21}\right) t+\cdots=0 .
\end{aligned}
$$

Next, equating the coefficients of (4.13) to zero, we obtain the following system of nonlinear algebraic equations

$$
\begin{aligned}
& t^{0}: y_{11}-2 y_{10} y_{20} z_{10} z_{20}=0, \\
& t^{1}:-2 y_{10} y_{20} z_{11} z_{20}+2 y_{12}-2 y_{10} y_{21} z_{10} z_{20}-2 y_{11} y_{20} z_{10} z_{20}
\end{aligned}
$$


$-2 y_{10} y_{20} z_{10} z_{21}=0$

$\vdots$
$t^{3}: \cdots$

$t^{0}: y_{21}+y_{10} y_{20} z_{20}^{2}=0$,

$t^{1}: y_{10} y_{21} z_{20}^{2}+2 y_{2} 2+2 y_{10} y_{20} z_{20} z_{21}+y_{11} y_{20} z_{20}^{2}=0$,

$t^{3}: \cdots$

$t^{0}: z_{11}-u_{0} y_{10} y_{20}-u_{0} z_{10} z_{20}=0$,

$t^{1}:-u_{0} y_{10} y_{21}-u_{1} z_{10} z_{20}-u_{0} y_{11} y_{20}-u_{0} z_{10} z_{21}-u_{0} z_{11} z_{20}$

$-u_{1} y_{10} y_{20}+2 z_{12}=0$

$\vdots$

$t^{4}: \cdots$

$t^{0}: y_{10} y_{20}^{2} z_{20}^{2} u_{0}+z_{21}=0$,

$t^{1}: 2 z_{22}+2 y_{10} y_{20}^{2} z_{20} z_{21} u_{0}+y_{11} y_{20}^{2} z_{20}^{2} u_{0}+y_{10} y_{20}^{2} z_{20}^{2} u_{1}$

$+2 y_{10} y_{20} y_{21} z_{20}^{2} u_{0}=0$,

$t^{4}: \cdots$

$t^{0}:-1+y_{10} y_{20}^{2}=0$,

$t^{1}: y_{11} y_{20}^{2}+2 y_{10} y_{20} y_{21}=0$,

$t^{2}: 2 y_{10} y_{20} y_{22}+y_{10} y_{21}^{2}+2 y_{11} y_{20} y_{21}+y_{12} y_{20}^{2}=0$,

$t^{3}: y_{11} y_{21}^{2}+2 y_{11} y_{20} y_{22}+2 y_{10} y_{20} y_{23}+2 y_{10} y_{21} y_{22}+2 y_{12} y_{20} y_{21}$

$+y_{13} y_{20}^{2}=0$,

$t^{4}: y_{1} 4 y_{20}^{2}+2 y_{11} y_{20} y_{23}+2 y_{13} y_{20} y_{21}+y_{12} y_{21}^{2}+2 y_{12} y_{20} y_{22}+2 y_{10} y_{21} y_{23}$

$+y_{10} y_{22}^{2}+2 y_{10} y_{20} y_{24}+2 y_{11} y_{21} y_{22}=0$. 
Now, in order to consider the initial condition of (4.11), we substitute them into (4.12) to obtain

$$
\begin{array}{rlrl}
y_{10} & =1, & y_{20} & =1, \\
z_{10} & =1, & z_{20}=1, \\
u_{0} & =1 . &
\end{array}
$$

It is important to notice that, from (4.14) and (4.15), we use only the powers $t^{i}$ $(i=0,1,2,3)$, because the rest of the information needed is taken from (4.19). From (4.11), we can observe that there is not an explicit equation for variable $u$. Instead, $u$ is implicitly included in equations for $z_{1}^{\prime}$ and $z_{2}^{\prime}$. Therefore, from (4.16) and (4.17), we use coefficients of powers $t^{i}(i=0,1,2,3,4)$ to collect enough information to compensate the presence of $u$ in those equations. Furthermore, powers $t^{i}(i=0,1,2)$ of (4.18) possesses redundant information that can be ignored. Hence, for (4.18), we can use only powers $t^{3}$ and $t^{4}$. Then, solving the NAEs composed by (4.14), (4.15), (4.16), (4.17), (4.18), and (4.19) results the following approximate solution

$$
\begin{aligned}
& y_{1}(t)=1+2 t+2 t^{2}+\frac{4}{3} t^{3}+\frac{2}{3} t^{4}, \\
& y_{2}(t)=1-t+\frac{1}{2} t^{2}-\frac{1}{6} t^{3}+\frac{1}{24} t^{4}, \\
& z_{1}(t)=1+2 t+2 t^{2}+\frac{4}{3} t^{3}+\frac{101}{174} t^{4}, \\
& z_{2}(t)=1-t+\frac{1}{2} t^{2}-\frac{1}{6} t^{3}+\frac{59}{696} t^{4}, \\
& u(t)=1+t+\frac{1}{2} t^{2}-\frac{1}{174} t^{3}-\frac{25}{58} t^{4} .
\end{aligned}
$$

Then, Laplace transformation is applied to (4.20) and then $1 / t$ is written in place of $s$. Afterwards, Padé approximant of order [2/2] is applied and $1 / s$ is written in place of $t$ for each variable. Finally, by using the inverse Laplace $s$ transformation, we obtain the exact solution for (4.11)

$$
\begin{aligned}
y_{1}(t) & =\exp (2 t), \\
y_{2}(t) & =\exp (-t), \\
z_{1}(t) & =\exp (2 t), \\
z_{2}(t) & =\exp (-t), \\
u(t) & =\exp (t) .
\end{aligned}
$$

\section{NUMERICAl SimUlation AND DisCUSSION}

Using LPSM method, we obtained the exact solution of two DAEs problems, one linear and other one nonlinear. It should be noticed that the high complexity of both problems was effectively handled by LPSM method due to the malleability of series 
method. Moreover, by inspection, redundant information in (4.7) and (4.18) was detected and neglected. Therefore, we obtained the series solutions (4.9) and (4.20). Next using the LP resummation method we obtained the exact solutions (4.10) and (4.21) for both case studies. The differential-algebraic problems are of relevance on several fields of sciences including microelectronics and chemistry. What is more, there is no any standard analytical method to solve this type of equations, converting the LPSM method into an attractive tool to solve DAEs problems. Finally, if the DAE problem do not possesses exact solution then the LP resummation method will provide a larger domain of convergence as reported in literature $[3,6,11,18,21,26-$ 28, 31,33].

On one side, semi-analytic methods like: homotopy perturbation method $[7,8$, 14, 16, 22, 23, 35, 41-43], homotopy analysis method[13, 15, 32], variational iteration method [1, 5, 20], among others [19], need an initial approximation for the sought solutions and the calculus of one or several adjustment parameters. If initial approximation is properly chosen the results can be highly accurate, nonetheless, no general method is available to choose such initial approximation. This issue motivates the use of adjustment parameters obtained by minimizing the least-squares error respect to the numerical solution. On the other side, series method or LPSM method do not need any trial equation as requirement for the method. Besides, series method obtain its coefficients using a straight forward procedure. Furthermore, at least for lower orders of approximation, the solution can be easily obtained using "solve" or "fsolve" commands of MAPLE or equivalent routines in Mathematica or MATLAB softwares. If high order approximations are required we propose the use of homotopy continuation methods $[4,12,24,25,29,30,36-40,44-46]$ by its well know ability to solve highly nonlinear equations.

\section{CONCLUSions}

This work presented LPSM method as a combination of the classic series method and a resummation method based on the Laplace and Padé transforms. By solving two problems, we presented the LPSM as a useful tool with high potential to solve linear/nonlinear differential-algebraic equations. The proposed method possesses a straightforward procedure, suitable for engineers and physicists. Finally, further research should be performed to solve other kind of related problems like fractional/partial nonlinear differential-algebraic equations, among others.

\section{ACKNOWLEDGEMENT}

The author would like to express his gratitude to Uriel Filobello-Nino, Roberto Castaneda-Sheissa, Rogelio-Alejandro Callejas-Molina, and Roberto Ruiz-Gomez for their contribution to this project. 


\section{REFERENCES}

[1] S. Abbasbandy, "A new application of he's variational iteration method for quadratic riccati differential equation by using adomian's polynomials," Journal of Computational and Applied Mathematics, vol. 207, no. 1, pp. 59 - 63, 2007, special Issue: Variational Iteration Method—Reality, Potential, and Challenges. [Online]. Available: http://www.sciencedirect.com/ science/article/pii/S0377042706004614

[2] S. Amat, M. J. Legaz, and P. Pedregal, "On a newton-type method for differential-algebraic equations," Journal of Applied Mathematics, pp. 1-15, 2012, doi:10.1155/2012/718608.

[3] D. Bahuguna, A. Ujlayan, and D. Pandey, "A comparative study of numerical methods for solving an integro-differential equation," Computers and Mathematics with Applications, vol. 57, no. 9, pp. 1485 - 1493, 2009.

[4] D. Bates, J. Hauenstein, A. Sommese, and C. Wampler, "Bertini: Software for numerical algebraic geometry," Available at http://www.nd.edu/ sommese/bertini.

[5] S. Chang, "A variational iteration method for solving troesch's problem," Journal of Computational and Applied Mathematics, vol. 234, no. 10, pp. 3043 - 3047, 2010.

[6] A. E. Ebaid, "A reliable aftertreatment for improving the differential transformation method and its application to nonlinear oscillators with fractional nonlinearities," Communications in Nonlinear Science and Numerical Simulation, vol. 16, no. 1, pp. 528 - 536, 2011.

[7] U. Filobello-Nino, H. Vazquez-Leal, , Y. Khan, R. Castaneda-Sheissa, A. Yildirim, L. HernandezMartinez, J. Sanchez-Orea, R. Castaneda-Sheissa, and F. R. Bernal, "Hpm applied to solve nonlinear circuits: a study case,” Appl. Math. Sci., vol. 6, no. 85-88, pp. 4331-4344, 2012.

[8] U. Filobello-Nino, H. Vazquez-Leal, R. Castaneda-Sheissa, A. Yildirim, L. Hernandez-Martinez, D. Pereyra-Diaz, A. Perez-Sesma, and C. Hoyos-Reyes, "An approximate solution of blasius equation by using hpm method," Asian Journal of Mathematics and Statistics, vol. 5, pp. 50-59, 2012, doi:103923/ajms.2012.50.59.

[9] A. Forsyth, "Theory of differential equations," Cambridge: University Press, pp. 78-90, 1906.

[10] K. Geddes, "Convergence behaviour of the newton iteration for first order differential equations," Proceedings of EUROSAM, pp. 189-199, 1979.

[11] A. Gökdoğan, M. Merdan, and A. Yildirim, "The modified algorithm for the differential transform method to solution of genesio systems," Communications in Nonlinear Science and Numerical Simulation, vol. 17, no. 1, pp. $45-51,2012$.

[12] T. Gunji, S. Kim, M. Kojima, A. Takeda, K. Fujisawa, and T. Mizutani, "Phom - a polyhedral homotopy continuation method for polynomial systems," Computing, vol. 73, p. 2004, 2003.

[13] H. Hassana and M. El-Tawil, "An efficient analytic approach for solving two-point nonlinear boundary value problems by homotopy analysis method," Mathematical methods in the applied sciences, vol. 34, pp. 977-989, 2011.

[14] J.-H. He, "Homotopy perturbation technique," Computer Methods in Applied Mechanics and Engineering, vol. 178, no. 3-4, pp. 257-262, 1999.

[15] J.-H. He, "Comparison of homotopy perturbation method and homotopy analysis method," Applied Mathematics and Computation, vol. 156, no. 2, pp. 527 - 539, 2004.

[16] J.-H. He, "An elementary introduction to the homotopy perturbation method," Computers and Mathematics with Applications, vol. 57, no. 3, pp. 410 - 412, 2009.

[17] E. Ince, “Ordinary differential equations,” Dover Publications, pp. 189-199, 1956.

[18] Y. Jiao, Y. Yamamoto, C. Dang, and Y. Hao, "An aftertreatment technique for improving the accuracy of adomian's decomposition method," Computers and Mathematics with Applications, vol. 43, no. 6-7, pp. 783-798, 2002. 
[19] Y. Khan, H. Vazquez-Leal, and N. Faraz, "An auxiliary parameter method using adomian polynomials and laplace transformation for nonlinear differential equations," Applied Mathematical Modelling, 2012, doi:10.1016/j.apm.2012.06.026.

[20] Y. Khan, H. Vazquez-Leal, L. Hernandez-Martinez, and N. Faraz, "Variational iteration algorithmii for solving linear and non-linear odes," International Journal of the Physical Sciences, vol. 7, no. 25, pp. 3099-4002, 2012.

[21] Y. Khan and N. Faraz, "Application of modified laplace decomposition method for solving boundary layer equation," Journal of King Saud University - Science, vol. 23, no. 1, pp. 115 - 119, 2011.

[22] Y. Khan, H. Vazquez-Leal, and L. Hernandez-Martinez, "Removal of noise oscillation term appearing in the nonlinear equation solution," Journal of Applied Mathematics, pp. 1-9, 2012, doi: $10.1155 / 2012 / 387365$.

[23] Y. Khan, H. Vazquez-Leal, and Q. Wu, "An efficient iterated method for mathematical biology model," Neural Computing and Applications, pp. 1-6, 2012, doi:10.1007/s00521-012-0952-z.

[24] T. L. Lee, T. Y. Li, and C. H. Tsai, "Hom4ps-2.0: a software package for solving polynomial systems by the polyhedral homotopy continuation method," Computing, vol. 83, no. 2-3, pp. 109_ 133, nov 2008.

[25] T.-Y. Li and C.-H. Tsai, "Hom4ps-2.0para: Parallelization of hom4ps-2.0 for solving polynomial systems," Parallel Comput., vol. 35, no. 4, pp. 226-238, apr 2009.

[26] M. Merdan, A. Gökdoğan, and A. Yildirim, "On the numerical solution of the model for hiv infection of cd $4^{+}$t cells," Computers and Mathematics with Applications, vol. 62, pp. $118-123$, 2011.

[27] S. Momani, G. Erjaee, and M. Alnasr, "The modified homotopy perturbation method for solving strongly nonlinear oscillators," Computers and Mathematics with Applications, vol. 58, no. 11-12, pp. $2209-2220,2009$.

[28] S. Momani and V. S. Ertürk, "Solutions of non-linear oscillators by the modified differential transform method," Computers and Mathematics with Applications, vol. 55, no. 4, pp. 833 - 842, 2008.

[29] A. Sarmiento-Reyes., R. Murphy-Arteaga., and H. Vazquez-Leal., "A maple-based homotopic circuit simulation package," A enviarse a IEEE Midwest Symposium on Circuits and Systems, Agosto 2001.

[30] A. J. Sommese and C. W. W. II, The Numerical Solution of Systems of Polynomials: Arising in Engineering And Science. World Scientific Pub Co Inc, 2005.

[31] N. Sweilam and M. Khader, "Exact solutions of some coupled nonlinear partial differential equations using the homotopy perturbation method," Computers and Mathematics with Applications, vol. 58, no. 11-12, pp. $2134-2141,2009$.

[32] Y. Tan and S. Abbasbandy, "Homotopy analysis method for quadratic riccati differential equation," Communications in Nonlinear Science and Numerical Simulation, vol. 13, no. 3, pp. $539-546$, 2008. [Online]. Available: http://www.sciencedirect.com/science/article/pii/S1007570406001353

[33] P.-Y. Tsai and C.-K. Chen, "An approximate analytic solution of the nonlinear riccati differential equation," Journal of the Franklin Institute, vol. 347, no. 10, pp. 1850 - 1862, 2010.

[34] S. K. Vanani and A. Aminataei, "Numerical solution of differential algebraic equations using a multiquadric approximation scheme," Mathematical and Computer Modelling, vol. 53, no. 5-6, pp. $659-666,2011$

[35] H. Vazquez-Leal, R. Castaneda-Sheissa, U. Filobello-Nino, A. Sarmiento-Reyes, and J. SanchezOrea, "High accurate simple approximation of normal distribution related integrals," Mathematical Problems in Engineering, vol. 2012, p. 22 pages, 2012, doi:10.1155/2012/124029.

[36] H. Vazquez-Leal, R. Castaneda-Sheissa, F. Rabago-Bernal, L. Hernandez-Martinez, A. SarmientoReyes, and U. Filobello-Nino, "Powering multiparameter homotopy-based simulation with a fast 
path-following technique," ISRN Applied Mathematics, vol. 2011, p. 7 pages, 2011, article ID 610637, doi:10.5402/2011/610637.

[37] H. Vazquez-Leal, R. Castaneda-Sheissa, A. Yildirim, Y. Khan, A. Sarmiento-Reyes, V. JimenezFernandez, A. Herrera-May, U. Filobello-Nino, F. Rabago-Bernal, and C. Hoyos-Reyes, "Biparameter homotopy-based direct current simulation of multistable circuits," British Journal of Mathematics \& Computer Science, vol. 2, no. 3, pp. 137-150, 2012, doi:10.5402/2011/610637.

[38] H. Vazquez-Leal, L. Hemandez-Martinez, A. Sarmiento-Reyes, and R. Castaneda-Sheissa, "Numerical continuation scheme for tracing the double bounded homotopy for analysing nonlinear circuits," in Proc. 2005 International Conference on Communications, Circuits and Systems, Hong Kong, China, 2005, pp. 1122-1126.

[39] H. Vazquez-Leal, L. Hernandez-Martinez, and A. Sarmiento-Reyes, "Double-bounded homotopy for analysing nonlinear resistive circuits," International Symposium on Circuits and Systems, vol. 4, pp. 3203-3206, May 2005.

[40] H. Vazquez-Leal, L. Hernandez-Martinez, A. Sarmiento-Reyes, R. Castaneda-Sheissa, and A. Gallardo-Del-Angel, "Homotopy method with a formal stop criterion applied to circuit simulation,” IEICE Electronic Express, vol. 8, no. 21, pp. 1808-1815, 2011, 10.1587/elex.8.1808.

[41] H. Vazquez-Leal, A. Sarmiento-Reyes, Y. Khan, U. Filobello-Nino, and A. Diaz-Sanchez, "Rational biparameter homotopy perturbation method and laplace-padé coupled version," Journal of Applied Mathematics, pp. 1-21, 2012, doi:10.1155/2012/923975.

[42] H. Vazquez-Leal, "Rational homotopy perturbation method," Journal of Applied Mathematics, pp. 1-14, 2012, doi:10.1155/2012/490342.

[43] H. Vazquez-Leal, U. Filobello-Nino, R. Castaneda-Sheissa, L. Hernandez-Martinez, and A. Sarmiento-Reyes, "Modified hpms inspired by homotopy continuation methods," Mathematical Problems in Engineering, vol. 2012, p. 19 pages, 2012, doi:10.1155/2012/309123.

[44] J. Verschelde, "Polynomial homotopy continuation with phepack," ACM Commun. Comput. Algebra, vol. 44, no. 3/4, pp. 217-220, jan 2011. [Online]. Available: http://doi.acm.org/10.1145/ 1940475.1940524

[45] L. T. Watson, S. C. Billups, and A. P. Morgan, "Algorithm 652: Hompack: a suite of codes for globally convergent homotopy algorithms," ACM Trans. Math. Softw., pp. 281-310, 1987.

[46] D. Wolf and S. R. Sanders, "Multiparameter homotopy methods for finding dc operating points of nonlinear circuits," IEEE Transactions on Circuits and Systems-I: Fundamental Theory and Applications, vol. 43, no. 10, pp. 824-837, October 1996.

\section{Author's address}

\section{Hector Vazquez-Leal}

Universidad Veracruzana, Electronic Instrumentation and Atmospheric Sciences School, Cto. Gonzalo Aguirre Beltrán S/N, 91000 Xalapa, Veracruz, México

E-mail address: hvazquez@uv.mx 